\title{
On a Difference Equation due to Stirling.
}

\author{
By Miss Eleanor Pairman.
}

(Read 11th January 1918. Received 17th January 1918.)

\section{Introduction.}

In 1730 there was published Stirling's Methodus Differentialis, and in it (Prop. VIII., p. 44) he considers the Difference Equation

$$
y(z)-\frac{z-m}{z} y(z+1)=\frac{1}{z-n},
$$

and shews that it is satisfied by an inverse factorial series $y_{1}(z)=\frac{1}{m}+\frac{n}{z} \frac{1}{m+1}+\frac{n(n+1)}{z(z+1)} \frac{1}{m+2}+\frac{n(n+1)(n+2)}{z(z+1)(z+2)} \frac{1}{m+3}+\ldots$

This result of Stirling's is the starting-point of the present paper : it will be convenient to modify his equations thus :

Taking

$$
\begin{aligned}
\frac{n-1}{z-1} y(z) & =u(z) \\
\text { and } \quad u(z+1, n+1) & =v(z)
\end{aligned}
$$

and making the necessary changes in (1) and (2), we obtain the result that the Difference Equation

$$
z v(z)-(z-m+1) v(z+1)=\frac{n}{z-n}
$$

is satisfied by

$$
v_{1}(z)=\frac{n}{z} \frac{1}{m}+\frac{n(n+1)}{z(z+1)} \frac{1}{m+1}+\frac{n(n+1)(n+2)}{z(z+1)(z+2)} \frac{1}{m+2}+\ldots
$$

We shall consider the difference equation in this latter form; its general solution is

$v(z)=C \frac{\Gamma(z)}{\Gamma(z-m+1)}-\frac{\Gamma(z)}{\Gamma(z-m+1)} \sum_{z} \frac{\Gamma(z-m+1)}{\Gamma(z+1)} \frac{n}{z-n}$,

where $\Sigma$, as usual, denotes the operation inverse to $\Delta$; and $C$ is a quantity independent of $z$. 
We shall prove that $v_{1}(z)$ and other solutions of the differenceequation (3) are also solutions of difference-equations with respect to the variables $m, n$, and we shall find relations connecting the various solutions.

\section{Difference Equation in $m$.}

From (5) we have

$$
v(z)=C \frac{\Gamma(z)}{\Gamma(z-m+1)}-\frac{\Gamma(z)}{\Gamma(z-m+1)} \sum_{z} \frac{\Gamma(z-m+1)}{\Gamma(z+m)} \frac{z}{z-n} .
$$

Let us now consider this as a function of $m$ and denote it by $\theta(m)$, supposing for the present that $C$ is independent of $m$, i.e. $\quad \theta(m)=C \frac{\Gamma(z)}{\Gamma(z-m+1)}-\frac{\Gamma(z)}{\Gamma(z-m+1)} \sum_{z} \frac{\Gamma(z-m+1)}{\Gamma(z+1)} \frac{n}{z-n}$.

Then

$$
\theta(m+1)=C \frac{\Gamma(z)}{\Gamma(z-m)}-\frac{\Gamma(z)}{\Gamma(z-m)} \sum_{z} \frac{\Gamma(z-m)}{\Gamma(z+m)} \frac{n}{z-n} .
$$

Therefore we have

$$
\begin{aligned}
& (z-m) \theta(m)-(n-m) \theta(m+1) \\
& =C \frac{\Gamma(z)}{\Gamma(z-m)}(1-n+m)-\frac{\Gamma(z)}{\Gamma(z-m)} \\
& \sum_{z}\left\{\frac{\Gamma(z-m+1)}{\Gamma(z+1)}-(n-m) \frac{\Gamma(z-m)}{\Gamma(z+1)}\right\} \frac{n}{z-n} \\
& \left.=C \frac{\Gamma(z)}{\Gamma(z-m)}(1-n+m)-\frac{\Gamma(z)}{\Gamma(z-m)} \sum_{z} \frac{\Gamma(z-m)}{\Gamma(z+1)} \overline{z-m}-\overline{n-m}\right\} \frac{n}{z-n} \\
& =C \frac{\Gamma(z)}{\Gamma(z-m)}(1-n+m)-\frac{n \Gamma(z)}{\Gamma(z-m)} \sum_{z} \frac{\Gamma(z-m)}{\Gamma(z+1)} \frac{z-n}{z-n} \\
& =C \frac{\Gamma(z)}{\Gamma(z-m)}(1-n+m)-\frac{\Gamma(z) n}{\Gamma(z-m)} \sum_{z} \frac{\Gamma(z-m)}{\Gamma(z-1)} .
\end{aligned}
$$

Now consider

$$
\begin{aligned}
\Delta \frac{\Gamma(z-m)}{\Gamma(z)} & =\frac{\Gamma(z-m+1)}{\Gamma(z+1)}-\frac{\Gamma(z-m)}{\Gamma(z)} \\
& =\frac{\Gamma(z-m)}{\Gamma(z+1)}(z-m-z) .
\end{aligned}
$$


Applying the operator $\$,

$\therefore$ we have

$$
\frac{\Gamma(z-m)}{\Gamma(z)}=-m \sum_{z} \frac{\Gamma(z-m)}{\Gamma(z+1)}
$$

$$
\begin{aligned}
(z- & m) \theta(m)-(n-m) \theta(m+1) \\
= & C \frac{\Gamma(z)}{\Gamma(z-m)}(1-n+m)+\frac{n}{m} \frac{\Gamma(z)}{\Gamma(z-m)} \frac{\Gamma(z-m)}{\Gamma(z)} \\
= & C \frac{\Gamma(z)}{\Gamma(z-m)}(1-n+m)+\frac{n}{m},
\end{aligned}
$$

which is a difference equation in $m$.

i.e. Every particular solution of the difference equation (3) in $z$ is a solution of the difference equation in $m$,

$$
(m-z) \theta(m)-(m-n) \theta(m+1)=-C \frac{\Gamma(z)}{\Gamma(z-m)}(1-n+m)-\frac{n}{m},
$$

where $C$ is the arbitrary constant in that particular solution.

We now require to find the difference equation in $m$ which is satisfied by the particular solution (4) of the original difference equation

$$
\begin{aligned}
& \theta(m)=v_{1}(z)=\frac{n}{z} \frac{1}{m}+\frac{n(n+1)}{z(z+1)} \frac{1}{m+1}+\frac{n(n+1)(n+2)}{z(z+1)(z+2)} \frac{1}{m+2}+\ldots \\
& \begin{aligned}
\therefore \theta(m+1)=\frac{n}{z} & \frac{1}{m+1}+\frac{n(n+1)}{z(z+1)} \frac{1}{m+2}+\frac{n(n+1)(n+2)}{z(z+1)(z+3)} \frac{1}{m+3}+\ldots \\
\therefore(m-z) \theta(m)= & \frac{n}{z}+\frac{n(n+1)}{z(z+1)}+\frac{n(n+1)(n+2)}{z(z+1)(z+2)}+\ldots \\
& -\frac{n}{m}-\frac{n(n+1)}{z} \frac{1}{m+1}-\frac{n(n+1)(n+2)}{z(z+1)} \frac{1}{m+2}-\ldots \\
\therefore(m-n) \theta(m+1) & =\frac{n}{z}+\frac{n(n+1)}{z(z+1)}+\ldots \\
& -\frac{n(n+1)}{z} \frac{1}{m+1}-\frac{n(n+1)(n+2)}{z(z+1)} \frac{1}{m+2}-\ldots
\end{aligned}
\end{aligned}
$$


Substituting in (6) we have

$$
\begin{aligned}
-C \frac{\Gamma(z)}{\Gamma(z-m)}(1-n+m)-\frac{n}{m} & =(m-z) \theta(m)-(m-n) \theta(m+1) \\
& =-\frac{n}{m}, \\
\therefore \quad C & =0,
\end{aligned}
$$

i.e. $\quad v_{1}(z)$ is a solution of the difference equation

$$
(m-z) \theta(m)-(m-n) \theta(m+1)=-\frac{n}{m} .
$$

3. Solution of the equation

$$
(m-z) \theta(m)-(m-n) \theta(m+1)=-\frac{n}{m} .
$$

Assume as a solution of this an inverse factorial series in $m$.

$$
\begin{aligned}
& \theta(m)=a_{0}+\frac{a_{1}}{m}+\frac{a_{3}}{m(m+1)}+\frac{a_{3}}{m(m+1)(m+2)}+\ldots \\
& \therefore \theta(m+1)=a_{0}+\frac{a_{1}}{m+1}+\frac{a_{2}}{(m+1)(m+2)}+\frac{a_{3}}{(m+1)(m+2)(m+3)}+\ldots \\
& \therefore(m-z) \theta(m)=(m-z) a_{0}+a_{1}+\frac{a_{2}}{m+1}+\frac{a_{3}}{(m+1)(m+2)}+\ldots \\
& -\frac{z a_{1}}{m}-\frac{z a_{2}}{m(m+1)}-\frac{z a_{2}}{m(m+1)(m+2)}-\cdots \\
& \therefore(m-n) \theta(m+1)=(m-n) a_{0}+a_{1}+\frac{a_{2}}{m+1}+\frac{a_{3}}{(m+1)(m+2)}+\ldots \\
& -\frac{(n+1) a_{1}}{m+1}-\frac{(n+2) a_{2}}{(m+1)(m+2)} \frac{(n+3) a_{3}}{(m+1)(m+2)(m+3)}+\ldots \\
& \therefore-\frac{n}{m}=(m-z) \theta(m)-(m-n) \theta(m+1) \\
& =(n-z) a_{0}+\left(\frac{n+1}{m+1}-\frac{z}{m}\right) a_{1}+\left(\frac{n+2}{m+2}-\frac{z}{m}\right) \frac{a_{2}}{m+1} \\
& +\left(\frac{n+3}{m+3}-\frac{z}{m}\right) \frac{a_{3}}{(m+1)(m+2)}+\ldots \\
& =(n-z) a_{0}+\frac{(n-z+1)(m+1)-(n+1)}{m(m+1)} a_{1}+\frac{(n-z+2)(m+2)-2(n+2)}{m(m+1)(m+2)} a_{2} \\
& +\frac{(n-z+3)(m+3)-3(n+3)}{m(m+1)(m+2)(m+3)} a_{3}+\ldots
\end{aligned}
$$




$$
\begin{aligned}
=(n-z) a_{0}+\frac{(n-z+1) a_{1}}{m}+\frac{(n-z+2) a_{2}-1(n+1) a_{1}}{m(m+1)} \\
\\
\quad+\frac{(n-z+3) a_{3}-2(n+2) a_{2}}{m(m+1)(m+2)}+\frac{(n-z+4) a_{4}-3(n+3) a_{3}}{m(m+1)(m+2)(m+3)}+\ldots .
\end{aligned}
$$

Equating coefficients of $\frac{1}{m}, \frac{1}{m(m+1)}, \ldots$, we obtain $a_{0}=0$

$$
a_{1}=-\frac{n}{n-z+1}
$$$$
a_{2}=\frac{1 \cdot(n+1)}{n-z+2} a_{1}=-\frac{1 \cdot n(n+1)}{(n-z+1)(n-z+2)}
$$$$
a_{3}=\frac{2(n+2)}{n-z+3} \quad a_{2}=-\frac{1.2 n}{(n-z+1)(n+z+2)(n-z-3)}
$$$$
a_{1}=\frac{3(n+3)}{n-z+4} a_{3}=-\frac{1 \cdot 2 \cdot 3 n(n+1)(n+2)(n+3)}{(n-z+1)(n-z+2)(n-z+3)(n-z+4)}
$$

\section{$\therefore \mathrm{We}$ have}

$$
\begin{aligned}
\theta_{1}(m)= & -\frac{n}{m} \frac{1}{n-z+1}-\frac{n(n+1)}{m(m+1)} \frac{1 !}{(n-z+1)(n-z+2)} \\
& -\frac{n(n+1)(n+2)}{m(m+1)(m+2)} \frac{2 !}{(n-z+1)(n-z+2)(n-z+3)}
\end{aligned}
$$

is a solution of the difference equation in $m$.

We can also find the general solution of the equation

$$
(m-z) \theta(m)-(m-n) \theta(m+1)=-\frac{n}{m} \text {. }
$$

First suppose that the term $-\frac{n}{m}$ is absent, then

$$
\theta(m+1)=\frac{m-z}{m-n} \theta(m),
$$

a solution of which is obviously

$$
\theta(m)=\alpha \frac{\Gamma(m-z)}{\Gamma(m-n)},
$$

where $\alpha$ is an arbitrary constant. 
Now assume

$$
\begin{array}{ll} 
& O(m)=u(m) \frac{\Gamma(m-z)}{\Gamma(m-n)} \\
\therefore \quad & \theta(m+1)=u(m+1) \frac{(m-z) \Gamma(m-z)}{(m-n) \Gamma(m-n)} \\
\therefore \quad & \frac{\Gamma(m-z+1)}{\Gamma(m-n)}\{u(m)-u(m+1)\}=-\frac{n}{m} \\
\therefore \quad & \Delta u(m)=\frac{n}{m} \frac{\Gamma(m-n)}{\Gamma(m-z+1)} \\
\therefore \quad & u(m)=C^{\prime}+\sum_{m} \frac{n}{m \Gamma(m-z+1)}
\end{array}
$$

where $C^{\prime}$ is an arbitrary constant.

$\therefore \quad \theta(m)=C^{\prime} \frac{\Gamma(m-z)}{\Gamma(m-n)}+\frac{\Gamma(m-z)}{\Gamma(m-n)} \sum_{m} \frac{n}{m} \frac{\Gamma(m-n)}{\Gamma(m-n+1)}, \ldots \ldots$

which is the general solution of the difference equation in $m$.

4. If now we consider $\theta(m)$, as given by (9), as a function of $z$, we can find a difference equation involving the arbitrary constant $C^{\prime}$ in $z$, which it must satisfy. Then, exactly as in $\S 2$, we find that the difference equation in $z$ which is satisfied by the series (8) is

$$
z v(z)-(z-m+1) v(z+1)=\frac{n}{z+n}
$$

which is the original difference equation again; i.e. $\theta_{1}(m)$ is a solution of both

$$
z v(z)-(z-m+1) v(z+1)=\frac{n}{z-n}
$$

and

$$
(m-z) \theta(m)-(m-n) \theta(m+1)=-\frac{n}{m} \text {. }
$$

$\therefore$ We see that both $v_{1}(z)$ and $\theta_{1}(m)$ are solutions.of each of the two equations

and

$$
\begin{aligned}
z v(z)-(z-m+1) v(z+1) & =\frac{n}{z-n} \\
(m-z) \theta(m)-(m-n) \theta m+1) & =-\frac{n}{m} .
\end{aligned}
$$


5. Difference Equation in $n$.

We have

$$
v(z)=C \frac{\Gamma(z)}{\Gamma(z-m+1)}-\frac{\Gamma(z)}{\Gamma(z-m-1)} \sum_{z} \frac{\Gamma(z-m+1)}{\Gamma(z+1)} \frac{n}{z-n} .
$$

Let us consider this as a function of $n$ and denote it by $\phi(n)$, assuming for the present that $C$ is independent of $n$, i.e. $\phi(n)=C \frac{\Gamma(z)}{\Gamma(z-m+1)}-\frac{\Gamma(z)}{\Gamma(z-n+1)} \sum_{z} \frac{\Gamma(z-m+1)}{\Gamma(z+1)} \frac{n}{z-n}$.

We could then find a difference equation involving $\phi(n)$, but it is simpler to deal with the function

$$
\psi(n)=\frac{\phi(n)}{n},
$$

i.e. $\quad \psi(n)=C \frac{1}{n} \frac{\Gamma(z)}{\Gamma(z-m+1)}-\frac{\Gamma(z)}{\Gamma(z-m+1)} \sum_{z} \frac{\Gamma(z-m+1)}{\Gamma(z+1)} \frac{1}{z-n}$.

$\therefore \psi(n+1)=\ell \frac{1}{1+n} \frac{\Gamma(z)}{\Gamma(z-m+1)} \frac{\Gamma(z)}{\Gamma(z-m+1)} \sum \frac{\Gamma(z-m+1)}{\Gamma(z+1)} \frac{1}{z-n-1}$

We shall now find a difference equation involving $\psi(n)$.

Consider

$$
\begin{gathered}
\Delta \frac{\Gamma(z-m+1)}{\Gamma(z+1)} \frac{1}{z-n-1} \\
=\frac{\Gamma(z-m+2)}{\Gamma(z+1)} \frac{1}{z-n}-\frac{\Gamma(z-m+1)}{\Gamma(z)} \frac{1}{z-n-1} \\
=\frac{\Gamma(z-m+1)}{\Gamma(z+1)}\left\{\frac{z-m+1}{z-n}-\frac{z}{z-n-1}\right\} \\
=\frac{\Gamma(z-m+1)}{\Gamma(z+1)}\left\{\frac{n-m+1}{z-n}-\frac{n+1}{z-n-1}\right\} . \\
\therefore \quad \frac{\Gamma(z-m+1)}{\Gamma(z)} \frac{1}{z-n-1}=\frac{\sum(z-m+1)}{\Gamma(z+1)}\left\{\frac{n-m+1}{z-n}-\frac{n+1}{z-n-1}\right\} \\
\therefore \quad \frac{\Gamma(z)}{z-n-1}=\frac{\sum(z-m+1)}{\Gamma(z-m+1)}\left\{\frac{n-m+1}{z-n}-\frac{n+1}{z-n-1}\right\} \\
=-(n-m+1) \psi(n)+\frac{n-m+1}{n} C \frac{\Gamma(z)}{\Gamma(z-m+1)} \\
+(n+1) \psi(n+1) \cdots C \frac{\Gamma(z)}{\Gamma(z-m+1} .
\end{gathered}
$$


$\therefore(n-m+1) \psi(n)-(n+1) \psi(n+1)=-\frac{m-1}{n} C \frac{\Gamma(z)}{\Gamma(z-m+1)}+\frac{1}{n-z-1}$

If now we wish to find the difference equation which is satisfied by the particular solution $v_{1}(z)$, we have only to substitute the series

$$
\frac{1}{z} \frac{1}{m}+\frac{n+1}{z(z+1)} \frac{1}{m+1}+\frac{(n+1)(n+2)}{z(z+1)(z+2)} \frac{1}{m+2}+\ldots
$$

for $\psi(n)$ in this equation, and we find inmediately that $C=0$.

Therefore the required difference equation in $n$ is

$$
(n-m+1) \psi(n)-(n+1) \psi(n+1)=\frac{1}{n-z+1} .
$$

\section{Solution of the equation}

$$
(n-m+1) \psi(n)-(n+1) \psi(n+1)=\frac{1}{n-z+1} .
$$

Assume as a solution a series of inverse factorials in $n-z+1$,

i.e. $\psi(n)=a_{0}+\frac{a_{1}}{n-z+1}+\frac{a_{2}}{(n-z+1)(n-z+2)}+\ldots$

$\therefore \psi(n+1)=a_{0}+\frac{a_{1}}{n-z+2}+\frac{a_{2}}{(n-z+2)(n-z+3)}+\ldots$

$\therefore \quad(n-m+1) \psi(n)$

$=(n+1) a_{0}+a_{1}+\frac{a_{2}}{n-z+2}+\frac{a_{3}}{(n-z+2)(n-z+3)}+\ldots$

$-m a_{0}+\frac{z-m}{n-z+1} a_{1}+\frac{(z-m) a_{2}}{(n-z+1)(n-z+2)}+\frac{(z-m)}{(n-z+1)(n-z+2)(n-z+3)}+\ldots$

$(n+1) \psi(n+1)$

$=(n+1) a_{0}+a_{1}+\frac{a_{3}}{n-z+2}+\frac{a_{3}}{(n-z+2)(n-z+3)}+\ldots$

$+\frac{(z-1) a_{1}}{n-z+2}+\frac{(z-2) a_{2}}{(n-z+2)(n-z+3)}+\frac{(z-3) a_{3}}{(n-z+2)(n-z+3)(n-z+4)}+\cdots$ 


$$
\begin{aligned}
& \therefore \quad \frac{1}{n-z+1}=(n-m+1) \psi(n)-(n+1) \psi(n+1) \\
& =-m a_{0}+\left(\frac{z-m}{n-z+1}-\frac{z-1}{n-z+2}\right) a_{1} \\
& +\left(\frac{z-m}{n-z+1}-\frac{z-2}{n-z+3}\right) \frac{a_{2}}{n-z+2} \\
& +\left(\frac{z-m}{n-z+1}-\frac{z-3}{n-z+4}\right) \frac{a_{3}}{(n-z+2)(n-z+3)} \\
& + \\
& =-m a_{0}+\frac{-(m-1)(n-z+2)+z-1}{(n-z+1)(n-z+2)} a_{1} \\
& +\frac{-(m-2)(n-z+3)+2(z-2)}{(n-z+1)(n-z+2)(n-z+3)} a_{2} \\
& +\frac{-(m-3)(n-z+4)+3(z-3)}{(n-z+1)(n-z+2)(n-z+3)(n-z+4)} a_{3}+\ldots \\
& =-m a_{0}-\frac{(m-1) a_{1}}{n-z+1}-\frac{(m-2) a_{2}-(z-1) a_{1}}{(n-z+1)(n-z+2)} \\
& -\frac{(m-3) a_{3}-2(z-2) a_{2}}{(n-z+1)(n-z+2)(n-z+3)} \\
& -\frac{(m-4) a_{4}-3(z-3)}{(n-z+1)(n-z+2)(n-z+3)(n-z+4)}+\ldots
\end{aligned}
$$

$\therefore \quad$ Equating coefficients of $\frac{1}{n-z+1}, \frac{1}{(n-z+1)(n-z+2)}, \ldots$, we have

$$
\begin{aligned}
& a_{0}=0 \\
& a_{1}=-\frac{1}{m-1} \\
& a_{2}=\frac{z-1}{m-2} a_{1}=-\frac{z-1}{(m-1)(m-2)} \\
& a_{3}=\frac{2(z-2)}{m-3} a_{2}=\frac{1 \cdot 2(z-1)(z-2)}{(m-1)(m-2)(m-3)} \\
& a_{4}=\frac{3(z-3)}{m-4} a_{3}=\frac{1 \cdot 2 \cdot 3 \cdot(z-1)(z-2)(z-3)}{(m-1)(m-2)(m-3)(m-4)}
\end{aligned}
$$




$$
\begin{aligned}
& \begin{aligned}
\therefore & \psi_{1}(n)=-\frac{1}{m-1} \frac{1}{n-z+1}-\frac{z-1}{(m-1)(m-2)} \frac{1 !}{(n-z+1)(n-z+2)} \\
& -\frac{(z-1)(z-2)}{(m-1)(m-2)(m-3)} \frac{2 !}{(n-z+1)(n-z+2)(n-z+3)} \\
- & \frac{(z-1)(z-2)(z-3)}{(m-1)(m-2)(m-3) m-4)} \frac{3 !}{(n-z+1)(n-z+2)(n-z+3)(n-z+4)} \\
\therefore & \phi_{1}(n)=n \psi_{1}(n) \\
= & -\frac{n}{m-1} \frac{1}{n-z+1}-\frac{n(z-1)}{(m-1)(m-2)} \frac{1 !}{(n-z+1)(n-z+2)} \\
& -\frac{n(z-1)(z-2)}{(m-1)(m-2)(m-3)} \frac{2 !}{(n-z+1)(n-z+2)(n-z+3)} \\
- & \frac{n(z-1)(z-2)(z-3)}{(m-1)(m-2)(m-3)(m-4)} \frac{3 !}{(n-z+1)(n-z+2)(n-z+3)(n-z+4)}
\end{aligned}
\end{aligned}
$$

To find the general solution of the equation we first assume that the term $\frac{1}{n-z+1}$ is absent,

then

a solution of which is

$$
\psi(n+1)=\frac{n-m+1}{n+1} \psi(n),
$$

$$
\psi(n)=\beta \frac{\Gamma(n-m+1)}{\Gamma(n+1)}
$$

where $\beta$ is an arbitrary constant.

$$
\begin{aligned}
& \text { Now assume that } \psi(n)=v(n) \frac{\Gamma(n-m+1)}{\Gamma(n+1)} \text {, } \\
& \therefore \psi(n+1)=v(n+1) \frac{(n-m+1) \Gamma(n-m+1)}{(n+1) \Gamma(n+1)} \text {. } \\
& \therefore \frac{1}{n-z+1}=(n-m+1) \psi(n)-(n+1) \psi(n+1) \\
& =\frac{\Gamma(n-m+2)}{\Gamma(n+1)}\{v(n)-v(n+1)\} \text {. } \\
& \therefore \quad \Delta v(n)=-\frac{\Gamma(n+1)}{\Gamma(n-m+2)} \frac{1}{n-z+1} \text {. } \\
& \therefore \quad v(n)=C^{\prime \prime}-\sum_{n} \frac{\Gamma(n+1)}{\Gamma(n-m+2)} \frac{1}{n-z+1}, \\
& \text { where } C^{\prime \prime} \text { is an arbitrary constant. }
\end{aligned}
$$




$$
\begin{aligned}
& \therefore \psi(n)=C^{\prime \prime} \frac{\Gamma(n-m+1)}{\Gamma(n+1)}-\frac{\Gamma(n-m+1)}{\Gamma(n+1)} \sum_{n} \frac{\Gamma(n+1)}{\Gamma(n-m+2)} \frac{1}{n-z+1} \\
& \therefore \phi(n)=n \psi(n) \\
& \quad C^{\prime \prime} \frac{\Gamma(n-m+1)}{\Gamma(n+1)}-\frac{\Gamma(n-m+1)}{\Gamma(n)} \sum_{n} \frac{\Gamma(n+1)}{\Gamma(n-m+2)} \frac{1}{n-z+1} \cdot(12)
\end{aligned}
$$

\section{Now}

$$
\begin{aligned}
\sum_{t} f(t) & =\text { constant }+f(t-1)+f(t-2)+f(t-3) \ldots \\
& =\text { constant }-f(t)-f(t+1)-f(t+2) \ldots \ldots .
\end{aligned}
$$

Therefore equation (5) gives

$$
\begin{gathered}
v(z)=C_{1} \frac{\Gamma(z)}{\Gamma(z-m+1)}-\frac{\Gamma(z) n}{\Gamma(z-m+1)}\left\{\frac{\Gamma(z-m)}{\Gamma(z)} \frac{1}{z-n-1}\right. \\
\left.\quad+\frac{\Gamma(z-m-1)}{\Gamma(z-1)} \frac{1}{z-n-2}+\frac{\Gamma(z-m-2)}{\Gamma(z-2)} \frac{1}{z-n-3}+\ldots\right\} \\
=C_{\mathrm{I}} \frac{\Gamma(z)}{\Gamma(z-m+1)}-\left\{\frac{1}{z-m} \frac{n}{z-n-1}+\frac{z-1}{(z-m)(z-m-1)} \frac{n}{z-n-2}\right. \\
\left.+\frac{(z-1)(z-2)}{(z-m)(z-m-1)(z-m-2)} \frac{n}{z-n-3}+\ldots\right\}
\end{gathered}
$$

and

$$
\begin{array}{r}
\begin{array}{r}
v(z)=C_{2} \\
\frac{\Gamma(z)}{\Gamma(z-m+1)}+\frac{n \Gamma(z)}{\Gamma(z-m+1)}\left\{\frac{\Gamma(z-m+1)}{\Gamma(z+1)} \frac{1}{z-n}\right. \\
\left.\quad+\frac{\Gamma(z-m+2)}{\Gamma(z+2)} \frac{1}{z-n+1}+\frac{\Gamma(z-m+3)}{\Gamma(z+3)} \frac{1}{z-n+2}+\ldots\right\} \\
=C_{2} \frac{\Gamma(z)}{\Gamma(z-m+1)}+\frac{1}{z} \frac{n}{z-n}+\frac{z-m+1}{z(z+1)} \frac{n}{z-n+1} \\
+\frac{(z-m+1)(z-m+2)}{z(z+1)(z+2)} \frac{n}{z-n+2}+\ldots .
\end{array}
\end{array}
$$

Also equation (9) gives$$
\theta(m)=C_{1}^{\prime} \frac{\Gamma(m-z)}{\Gamma(m-n)}+\frac{\Gamma(m-z)}{\Gamma(m-n)} n\left\{\frac{\Gamma(m-n-1)}{\Gamma(m-z)} \frac{1}{m-1}\right.
$$$$
\left.+\frac{\Gamma(m-n-2)}{\Gamma(m-z-1)} \frac{1}{m-2}+\frac{\Gamma(m-n-3)}{\Gamma(m-z-2)} \frac{1}{m-3}+\ldots\right\}
$$$$
=C_{1}{ }^{\prime} \frac{\Gamma(m-z)}{\Gamma(m-n)}+\frac{1}{m-n-1} \frac{n}{m-1}+\frac{(m-z-1)}{(m-n-1)(m-n-2)} \frac{n}{m-2}
$$$$
\left.+\frac{(m-z-1)(m-z-2)}{(m-n-1)(m-. n-2)(m-n-3)} \frac{n}{m-3}+\ldots . .\right)
$$ 
and

$$
\begin{aligned}
\theta(m)= & C_{2}^{\prime} \frac{\Gamma(m-z)}{\Gamma(m-n)}-\frac{\Gamma(m-z)}{\Gamma(m-n)} n\left\{\frac{\Gamma(m-n)}{\Gamma(m-z+1)} \frac{1}{m}\right. \\
& \left.\quad+\frac{\Gamma(m-n+1)}{\Gamma(m-z+2)} \frac{1}{m+1}+\frac{\Gamma(m-n+2)}{\Gamma(m-z+3)} \frac{1}{m+2}+\ldots . .\right\} \\
= & C_{2}^{\prime} \frac{\Gamma(m-z)}{\Gamma(m-n)}-\left\{\frac{1}{m-z} \frac{n}{m}+\frac{m-n}{(m-z)(m-z+1)} \frac{n}{m+1}\right. \\
& \left.\quad+\frac{(m-n)(m-n+1)}{(m-z)(m-z+1)(m-z+2)} \frac{n}{m+2}+\ldots \ldots\right\}
\end{aligned}
$$

Also equation (12) gives

$$
\begin{aligned}
\phi(n)=C_{\mathrm{i}}^{\prime \prime} & \frac{\Gamma(n-m+1)}{\Gamma(n)}-\frac{\Gamma(n-m+1)}{\Gamma(n)}\left\{\frac{\Gamma(n)}{\Gamma(n-m+1)} \frac{1}{n-z}\right. \\
& \left.+\frac{\Gamma(n+1)}{\Gamma(n-m)} \frac{1}{n-z-1}+\frac{\Gamma(n-2)}{\Gamma(n-m-1)} \frac{1}{n-z-2}+\ldots \ldots\right\} \\
= & C_{1}^{\prime \prime} \frac{\Gamma(n-m+1)}{\Gamma(n)}-\left\{\frac{1}{n-z}+\frac{n-m}{n-1} \frac{1}{n-z-1}\right. \\
& \left.+\frac{(n-m)(n-m-1)}{(n-1)(n-2)} \frac{1}{n-z-2}+\ldots \ldots\right\}
\end{aligned}
$$

and

$$
\begin{array}{r}
\phi(n)=C_{2}^{\prime \prime} \frac{\Gamma(n-m+1)}{\Gamma(n)}+\frac{\Gamma(n-m+1)}{\Gamma(n)}\left\{\frac{\Gamma(n+1)}{\Gamma(n-m+2)} \frac{1}{n-z+1}\right. \\
\left.\quad+\frac{\Gamma(n+2)}{\Gamma(n-m+3)} \frac{1}{n-z+2}+\frac{\Gamma(n+3)}{\Gamma(n-m+4)} \frac{1}{n-z+3}+\ldots \ldots\right\} \\
=C_{2}^{\prime \prime} \frac{\Gamma(n-m+1)}{\Gamma(n)}+\frac{n}{n-m+1} \frac{1}{n-z+1}+\frac{n(n+1)}{(n-m+1)(n-m+2)} \frac{1}{n-z+2} \\
\quad+\frac{n(n+1)(n+2)}{(n-m+1)(n-m+2)(n-m+3)} \frac{1}{n-z+3}+\ldots \ldots .
\end{array}
$$

Dropping the arbitrary constant in each case, we obtain from these and the series $4,8,11$, the following nine series:- 
욜

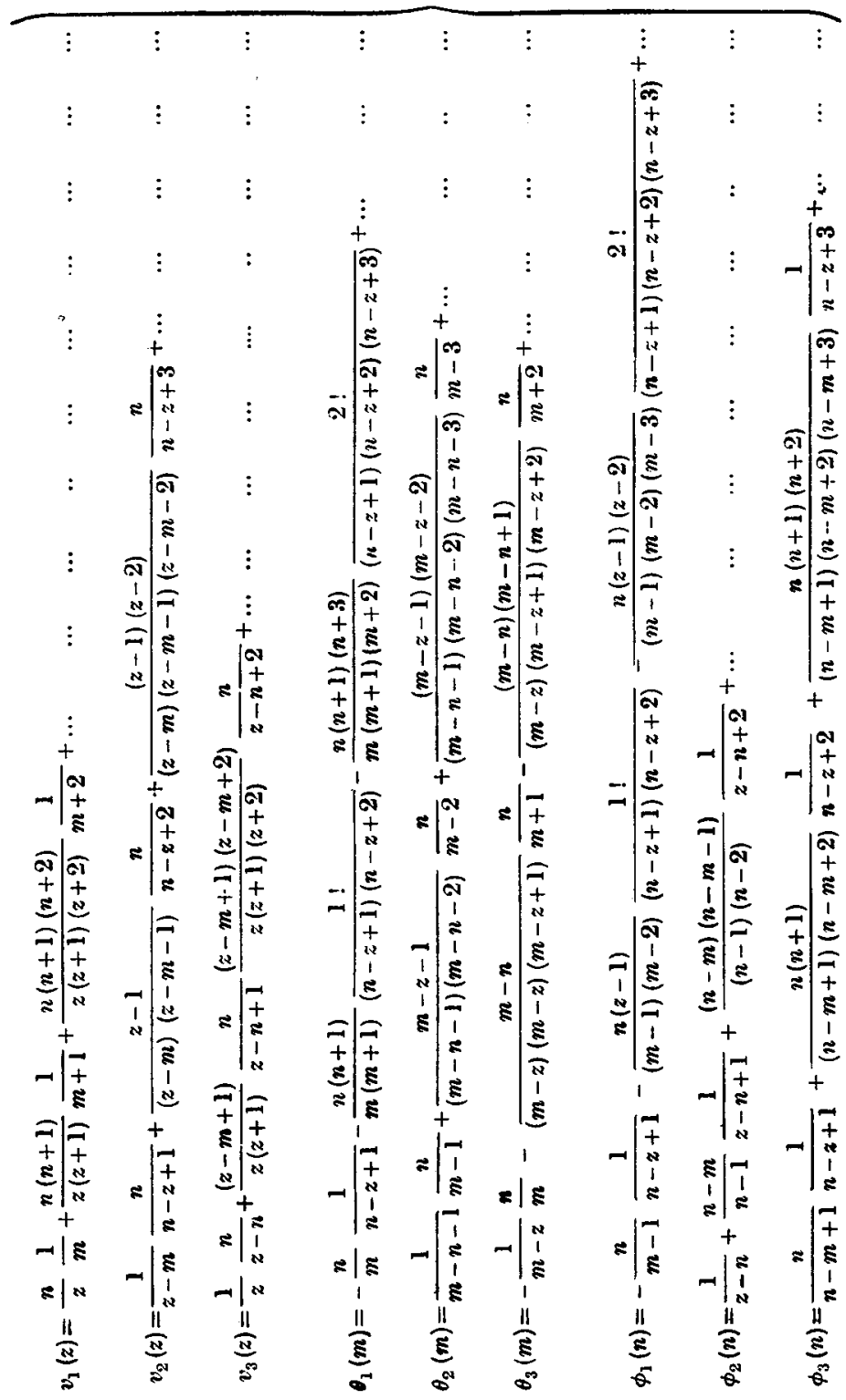


8. Equality of certain series.

Now denote $v_{1}(z)$ by $v(z, m, n)$.

$$
\begin{aligned}
\therefore \quad v_{2}(z) & =\frac{n}{z} v(m-z, n-z+1,-z) \\
v_{3}(z) & =\frac{n}{z-m} v(z, z-n, z-m) \\
\theta_{2}(z) & =\frac{n}{z-m} v(n-m+1,1-m, z-m) \\
\theta_{3}(z) & =\frac{-n}{m-n-1} v(m-z, m, m-n-1) \\
\phi_{2}(z) & =\frac{n}{n-m+1} v(-n, z-n, m-n-1) \\
\phi_{3}(z) & =v(n-m+1, n-z+1, n) .
\end{aligned}
$$

Now it was proved by Stirling that $v_{1}=v_{i j}$,

$$
\begin{aligned}
& \quad \text { i.e. } \quad v(z, m, n)=\frac{n}{z-m} v(z, z-n, z-m) \\
& \therefore \quad v(m-z, n-z+1,-z)=\frac{-z}{m-n-1} v(m-z, m, m-n-1) \\
& \therefore \quad \frac{n}{z} v(m-z, n-z+1,-z)=\frac{-n}{m-n-1} v(u-z, m, m-n-1), \\
& \quad \text { i.e. } \quad v_{2}=\theta_{3} .
\end{aligned}
$$

Also

$$
\begin{aligned}
& \quad v(n-m+1,1-m, z-m)=\frac{z-m}{n} v(n-m+1, n-z+1, n) \\
& \therefore \frac{n}{z-m} v(n-m+1,1-m, z-m)=v(n-m+1, n-z+1, n), \\
& \quad \text { i.e. } \theta_{2}=\phi_{3} . \\
& \text { Also } \\
& \quad v(-n, z-n, m-n-1)=\frac{m-n-1}{-z} v(-n, 1-m,-z) \\
& \therefore \quad \frac{n}{n-m+1} v(-n, z-n, m-n-1)=\frac{n}{z} v(-n, 1-m,-z), \\
& =\frac{n}{z}\left\{\frac{-z}{-n} \frac{1}{1-m}+\frac{-z(1-z)}{-n(1-) n} \frac{1}{2-m}+\frac{-z(1-z)(2-z)}{-n(1-n)(2-n)} \frac{1}{3-m}+\ldots\right\} \\
& =-\frac{1}{m-1}-\frac{z-1}{n-1} \frac{1}{m-2}-\frac{(z-1)(z-2)}{(n-1)(n-2)} \frac{1}{m-2}-\ldots,
\end{aligned}
$$

which connects certain of the series. 
9. Relation between the series.

$v_{1}(z)$ and $v_{2}(z)$ are both solutions of the difference equation in $\approx$.

Therefore they are both particular cases of the general solution

$$
v(z)=C \frac{\Gamma(z)}{\Gamma(z-m+1)}-\frac{\Gamma(z)}{\Gamma(z-m+1)} \sum \frac{\Gamma(z-m+1)}{\Gamma(z+1)} \frac{n}{z-n} .
$$

Therefore their difference must be of the form

$$
C \frac{\Gamma(z)}{\Gamma(z-m+1)} \text {. }
$$

Up till now we have assumed $C$ to be an arbitrary constant, but it may also be a periodic function of $\approx$.

For, suppose it involves $\approx$.

$$
\begin{aligned}
v(z)= & C(z) \frac{\Gamma^{\prime}(z)}{\Gamma(z-m+1)}-\frac{\Gamma^{\prime}(z)}{\Gamma^{\prime}(z-m+1)} \sum \frac{\Gamma^{\prime}(z-m+1)}{\Gamma(z+1)} \frac{n}{z-n} \\
r(z+1)= & C(z+1) \frac{\Gamma(z+1)}{\Gamma(z-m+2)} \frac{\Gamma^{\prime}(z+1)}{\Gamma^{\prime}(z-m+2)} \sum \frac{\Gamma(z-m+2)}{\Gamma(z+2)} \frac{n}{z-n+1} \\
\therefore \quad z v(z)- & (z-m+1) v(z+1) \\
= & \frac{\Gamma(z+1)}{\Gamma(z-m+1)}\{C(z)-C(z+1)\}+\frac{n}{z-n} .
\end{aligned}
$$

Therefore, in order that the difference equation may be satisfied, we must have

$$
C(z)-C(z+1) \equiv 0,
$$

which is true, provided $C(z)$ is a periodic function of $z$.

$$
\therefore \quad v_{1}-v_{z}=C \frac{\Gamma(z)}{\Gamma(z-m+1)},
$$

where $C$ is either a constant or a periodic function of $z$.

But $v_{2}=\theta_{i ;}$.

Therefore $v_{2}$ is a solution of the difference equation in $m$, of which $v_{1}$ is also a solution.

$$
\therefore v_{1}-v_{2}=C^{\prime} \frac{\Gamma(m-z)}{\Gamma(m-n)},
$$

where $C$ does not involve $m$, or else is a periodic function of $m$.

Also, $v_{2}$ is a solution of the difference equation in $n$.

For, if $\psi(n)=\frac{1}{z-m} \frac{1}{n-z+1}+\frac{z-1}{(z-m)(z-m-1)} \frac{1}{n-z+2}+\ldots$ 
then $(n-m+1) \psi(n)-(n+1) \psi(n+1)$

$$
\begin{aligned}
& =\frac{1}{z-m}\left(\frac{n-m+1}{n-z+1}-\frac{n+1}{n-z+2}\right)+\frac{(z-1)}{(z-m)(z-m-1)}\left(\frac{n-m+1}{n-z+2}-\frac{n+1}{n-z+3}\right) \\
& +\frac{(z-1)(z-2)}{(z-m)(z-m-1)(z-m-2)}\left(\frac{n-m+1}{n-z+3}-\frac{n+1}{n-z+4}\right)+\ldots \\
& =\frac{1}{z-m}\left(\frac{z-m}{n-z+1}-\frac{z-1}{n-z+2}\right)+\frac{z-1}{(z-m)(z-m-1)}\left(\frac{z-m-1}{n-z+2}-\frac{z-2}{n-z+3}\right) \\
& +\frac{(z-1)(z-2)}{(z-m)(z-m-1)(z-n-2)}\left(\frac{z-m-2}{n-z+3}-\frac{z-3}{n-z+4}\right)+\ldots \\
& =\frac{1}{u-z+1}-\frac{z-1}{z-m} \frac{1}{n-z+2}+\frac{z-1}{z-m} \frac{1}{n-z+2}-\frac{(z-1)(z-2)}{(z-m)(z-m-1)} \frac{1}{n-z+3} \\
& +\frac{(z-1)(z-2)}{(z-m)(z-m-1)} \frac{1}{n-z+3}-\ldots \\
& =\frac{1}{n-z+1} \text {, }
\end{aligned}
$$

and $v_{1}$ is also a solution of the same equation.

$$
\therefore v_{1}-v_{2}=C^{\prime \prime} \frac{\Gamma(n-m+1)}{\Gamma(n)},
$$

where $C^{\prime \prime}$ is either independent of $n$ or else is a periodic function of $n$.

Therefore, combining these three results (14), (15), (16), we have

$$
\begin{aligned}
C \frac{\Gamma(z)}{\Gamma(z-m+1)} & =C^{\prime} \frac{\Gamma(m-z)}{\Gamma(m-n)}=C^{\prime \prime} \frac{\Gamma(n-m+1)}{\Gamma(n)} . \\
\therefore \quad C^{\prime} & =\frac{\Gamma(z) \Gamma(m-n)}{\Gamma(z-m+1) \Gamma(m-z)} C^{\prime} \\
& =\Gamma(z) \Gamma(m-n) \frac{\sin \pi(m-z)}{\pi} C .
\end{aligned}
$$

Now $C^{\prime \prime}$ is periodic in $m$.

$$
\therefore C=\Gamma(n-m+1) \beta
$$

where $\beta$ is periodic in $m$; for, if so, then

$$
\begin{aligned}
C^{\prime} & =\Gamma^{\prime}(z) \Gamma^{\prime}(m-n) \Gamma^{\prime}(n-m+1) \frac{\sin \pi(m-z)}{\pi} \beta \\
& =\Gamma(z) \frac{\sin \pi(m-z)}{\sin \pi(m-n)} \beta,
\end{aligned}
$$

which is periodic in $m$. 
Also

$$
\begin{aligned}
C^{\prime \prime} & =\frac{\Gamma(z) \Gamma(n)}{\Gamma(z-m+1) \Gamma(n-m+1)} C \\
& =\frac{\Gamma(z) \Gamma(n)}{\Gamma(z-m+1)} \beta .
\end{aligned}
$$

$C^{\prime \prime}$ is periodic in $n$.

$$
\therefore \beta=\frac{1}{\Gamma(n)} \alpha,
$$

where $\alpha$ is periodic in $n$; for, if so, then

which is periodic in $n$.

$$
C^{\prime \prime}=\frac{\Gamma(z)}{\Gamma(z-m+1)} \alpha,
$$

where $\alpha$ is periodic in $z, m, n$.

$$
\therefore \quad C=\frac{\Gamma(n-m+1)}{\Gamma(n)} \alpha
$$

$$
\therefore \quad v_{1}-v_{z}=\alpha(z, m, n) \frac{\Gamma(z) \Gamma(n-m+1)}{\Gamma(n) \Gamma(z-m+1)}
$$

where $\alpha$ is a periodic function of its three arguments.

If the value of $\alpha$ is known for any particular value $z_{1}$ of the argument $z$, we can at once write down the relation between $v_{1}$ and $v_{2}$ for values of the argument

$$
\begin{aligned}
& z_{1}+1, z_{1}+2, z_{1}+3, z_{1}+4,+\ldots, \\
& z_{1}-1, z_{1}-2, z_{1}-3 \ldots .
\end{aligned}
$$

In exactly the same way we may shew that the difference of any two of the series must be of the same form.

10. Some particular cases.

Stirling, in the work already referred to, points out that if $n$ is a negative integer, the series $v_{1}$ gives the sum of the series $v_{3}$ exactly.

We will now shew that if $z$ is a positive integer, we can find sum of the series $v_{1}$ by means of the hypergeometric function.

First, let $z=1$.

Then

$$
\begin{aligned}
v_{1}(z)= & \frac{n}{1} \frac{1}{m}+\frac{n(n+1)}{1.2} \frac{1}{m+1}+\frac{n(n+1)(n+2)}{1.2 .3} \frac{1}{m+2}+\ldots \\
=\frac{1}{m-1}\left\{\frac{n}{1} \frac{m-1}{m}+\right. & \frac{n(n+1)}{1.2} \frac{(m-1) m}{1.2 .3} \\
& \left.+\frac{n(n+1)(n+2)}{1.2 .3} \frac{(m-1) m(m+1)}{m(m+1)(m+2)}+\ldots\right\}
\end{aligned}
$$




$$
=\frac{1}{m-1}\{F(n, m-1, m, 1)-1\}
$$

where $F$ is the hypergeometric function,

$$
\begin{aligned}
& =\frac{1}{m-1}\left\{\frac{\Gamma(m) \Gamma(1-n)}{\Gamma(1) \Gamma(m-n)}-1\right\} \\
& =\frac{1}{1-m}+\frac{\dot{\Gamma(m-1) \Gamma(1-n)}}{\Gamma(m-n)} .
\end{aligned}
$$

Also

$$
v_{:}(z)=\frac{1}{1-m} \frac{n}{n} \text {. }
$$

$\therefore v_{1}(z)-v_{2}(z)=\frac{\Gamma(m-1) \Gamma(1-n)}{\Gamma(m-n)}$.

$\therefore$ For $z=1$,

$$
\alpha(z, m, n)=\frac{\Gamma(z) \Gamma(n-m+1)}{\Gamma(n) \Gamma(z-m+1)}=\frac{\Gamma(m-1) \Gamma(1-n)}{\Gamma(m-n)},
$$

i.e. $\quad \alpha \frac{\Gamma(1) \Gamma(n-m+1)}{\Gamma(n) \Gamma(2-m)}=\frac{\Gamma(m-1) \Gamma(1-u)}{\Gamma(m-n)}$,

i.e. $\quad \alpha=\frac{\Gamma(m-1) \Gamma(1-n) \Gamma(n) \Gamma(2-m)}{\Gamma(m-n) \Gamma(n-m+1)}$.

$$
\begin{aligned}
& =\frac{\pi \sin (m-n) \pi}{\sin n \pi \sin (m-1) \pi} \\
& =\frac{-\pi \sin (m-n) \pi}{\sin m \pi \sin n \pi},
\end{aligned}
$$

which can be easily calculated from a table of sines.

Now $\alpha$ is unaltered if $z$ be replaced by $z+1$; i.e. $\alpha$ has this value for all positive integral values of $z$.

i.e. If $z$ is a positive integer, then

$$
\begin{aligned}
& \frac{n}{z} \frac{1}{m}+\frac{n(n+1)}{z(z+1)} \frac{1}{m+1}+\frac{n(n+1)(n+2)}{z(z+1)(z+2)} \frac{1}{m+2}+\ldots \\
& =\frac{-\pi \sin (m-n) \pi}{\sin m \pi \sin n \pi} \quad \frac{\Gamma(z) \Gamma(n-m+1)}{\Gamma(n) \Gamma(z-m+1)}+v_{2} \\
& =\frac{-\pi}{\sin m \pi} \frac{\Gamma(z)}{\Gamma(z-m+1)} \frac{\Gamma(1-n)}{\Gamma(m-n)}+\frac{1}{z-m} \frac{n}{n-z+1} \\
& \quad+\frac{z-1}{(z-m)(z-m-1)} \frac{n}{n-z+2}
\end{aligned}
$$




$$
\begin{aligned}
& +\frac{(z-1)(z-2)}{(z-m)(z-m-1)(z-m-2)} \frac{n}{n-z+3}+\ldots \\
& +\frac{(z-1)(z-2) \ldots 2.1}{(z-m)(z-m-1) \ldots(1-m)} \frac{n}{n} \ldots \ldots \ldots \ldots \ldots
\end{aligned}
$$

which contains only a finite number of terms.

Again, if $m=z+1$,

$$
\begin{aligned}
v_{1} & =\frac{n}{z(z+1)}+\frac{n(n+1)}{z(z+1)(z+2)}+\ldots \\
& =\frac{n}{z}\left\{\frac{1}{z+1}+\frac{n+1}{(z+1)(z+2)}+\ldots\right\} \\
& =\frac{n}{z} \frac{1}{z-n} . \\
v_{2} & =-n\left\{\frac{1}{n-z+1}+\frac{1-z}{12} \frac{1}{n-z+2}+\frac{(1-z)(2-z)}{1.2 .3} \frac{1}{n-z+3}+\ldots\right\} \\
& =\frac{n}{z(n-z)}\left\{1+\frac{-z}{1} \frac{n-z}{n-z+1}+\frac{-z(1-z)}{1 \cdot 2} \frac{(n-z)(n-z+1)}{(n-z+1)(n-z+2)}+\ldots-1\right\} \\
& =\frac{n}{z(n-z)}\{F(-z, n-z, n-z+1,1)-1\} \\
& =\frac{n}{z(n-z)} \frac{\Gamma(n-z+1) \Gamma(1+z)}{\Gamma(1) \Gamma(1+n)}+\frac{n}{z(z-n)} \\
& =\frac{F(n-z) \Gamma}{\Gamma(n)} \frac{n}{z(z-n)}+\quad \ldots \quad v_{2}-v_{1}=\frac{\Gamma(n-z) \Gamma(z)}{\Gamma(n)} \text { for } m=z+1 .
\end{aligned}
$$

But

where $C^{\prime}$ is periodic in $m$

$$
v_{2}-v_{1}=C^{\prime \prime} \frac{\Gamma(m-z)}{\Gamma(m-n)}
$$

$$
\begin{aligned}
& \therefore \quad \frac{\Gamma(n-z) \Gamma(z)}{\Gamma(n)}=C^{\prime} \frac{\Gamma(1)}{\Gamma(z-n+1)} \\
& \therefore \quad C^{\prime}=\Gamma(z-n+1) \Gamma(n-z) \frac{\Gamma(z)}{\Gamma(n)} \\
& \text { for } m-z=1,
\end{aligned}
$$

and therefore for $m-z=$ any positive integer; 
i.e. If $m-z$ is a positive integer, then

$$
\begin{aligned}
v_{3} & =v_{1}+\frac{\Gamma(z-n+1) \Gamma(n-z) \Gamma(z) \Gamma(m-z)}{\Gamma(m-n) \Gamma(n)} \\
& =v_{3}+\frac{\Gamma(z) \Gamma(m-z)}{\Gamma(n) \Gamma(m-n)} \frac{\pi}{\sin \pi(n-z)}
\end{aligned}
$$

i.e. $\frac{1}{z-m} \frac{n}{n-z+1}+\frac{z-1}{(z-n i)(z-m-1)} \frac{n}{n-z+2}$

$$
\begin{aligned}
& \quad+\frac{(z-1)(z-2)}{(z-m)(z-m-1)(z-m-2)} \frac{n}{n-z+3}+\ldots \\
& =\frac{\Gamma(z)}{\Gamma(n)} \frac{\Gamma(m-z)}{\Gamma(m-n)} \frac{\pi}{\sin \pi(n-z)}+\frac{1}{z} \frac{n}{z-n}+\frac{z-m+1}{z(z+1)} \frac{n}{z-n+1} \\
& \quad+\frac{(z-m+1)(z-m+2)}{z(z+1)(z+2)} \frac{n}{z-n+2}+\ldots \\
& \quad+\frac{(z-m+1)(z-m+2) \ldots(-2)(-1)}{z(z+1)-\ldots-(m-1)} \frac{n}{m-n-1} \cdot \ldots \ldots \text { (19) }
\end{aligned}
$$

In exactly the same way, if $n-m$ is a positive integer, we can calculate $v_{1}$ by means of $\theta_{3}$, using the fact that

where $C^{\prime \prime}$ is a periodic function of $n$.

$$
v_{1}-v_{3}=C^{\prime \prime} \frac{\Gamma(n-m+1)}{\Gamma(n)}
$$

11. By giving various values to $z, m, n$ in the series which are equal to one another, we obtain identities in $z$.

e.g. if in $v_{1}$ and $v_{3}$ we put

we find the identity

$$
\begin{aligned}
& m=z \\
& m=z-1
\end{aligned}
$$

$$
\begin{gathered}
(z-1)\left\{\frac{1}{z^{2}}+\frac{1}{(z+1)^{2}}+\frac{1}{(z+2)^{2}}+\ldots\right\} \\
=(z-1)\left\{\frac{1}{z}+\frac{1}{z(z+1)^{2}}+\frac{1}{z(z+1)(z+2)} \frac{1.2}{3}\right. \\
\left.\quad+\frac{1}{z(z+1)(z+2)(z+3)} \frac{1.2 .3}{4}+\ldots\right\} \\
\quad \therefore \frac{1}{z^{2}}+\frac{1}{(z+1)^{2}}+\frac{1}{(z+2)^{2}}+\ldots \\
=\frac{1}{z}+\frac{1}{z(z+1)} \frac{1}{2}+\frac{1}{z(z+1)(z+2)} \frac{1.2}{3}+\ldots \ldots \ldots \ldots(20)
\end{gathered}
$$


Now, put $z=14$ and we find

$$
\frac{1}{14^{2}}+\frac{1}{15^{2}}+\ldots=074040270 \text {, }
$$

only 13 terms being required for its computation. But we find from Barlow's tables that

$$
\begin{gathered}
\frac{1}{1^{2}}+\frac{1}{2^{2}}+\frac{1}{3^{2}}+\ldots \frac{1}{13^{2}}=1.570893798 . \\
\therefore \quad \frac{1}{1^{2}}+\frac{1}{2^{2}}+\frac{1}{3^{2}}+\ldots \\
=\quad 1.570893798 \\
\quad+\quad+074040270 \\
=\quad 1.64493407
\end{gathered}
$$

which gives the sums of the squares of the reciprocals of the natural numbers - a series which, in its original form, is only very slowly convergent. 